\title{
El largo camino hacia la economía lícita: Estado y estrategias de desarrollo alternativo en el «milagro de San Martín» ${ }^{+}$
}

\author{
HERNÁN MANRIQUE * \\ Pontificia Universidad Católica del Perú \\ hernan.manrique@pucp.pe \\ https://doi.org/10.18800/rcpg.201701.007
}

\section{RESUMEN}

El presente artículo analiza el caso de desarrollo alternativo de la región San Martín, conocido por diversas agencias de lucha contra las drogas como el «milagro de San Martín». En primer lugar, se presentan los elementos teóricos del estudio, donde las capacidades del Estado y el control territorial se constituyen como determinantes para el éxito del desarrollo alternativo. En base a estas consideraciones, el artículo establece tres periodos diferenciados de implementación del desarrollo alternativo en la región San Martín. En el primero de ellos, de 1981 a 1989, el auge del narcotráfico y la presencia subversiva dificultaron el desenvolvimiento de los programas de desarrollo alternativo. En el segundo periodo, de 1990 a 2002, la caída del precio de la coca, la expansión del hongo Fusarium oxysporum, la suspensión de la erradicación forzada de coca y el debilitamiento de las organizaciones subversivas abrieron una ventana de oportunidad para la implementación del desarrollo alternativo. En el tercer y último periodo, de 2003 a 2015, la participación de las autoridades políticas en las decisiones del desarrollo alternativo y el contexto internacional favorable de los precios de los cultivos alternativos ayudaron a sentar las bases del «modelo definitivo» de desarrollo alternativo de San Martín.

Palabras clave: crimen, economía agraria, política interna, desarrollo económico y social, ayuda al desarrollo.

The long road to the legal economy: State and strategies of alternative development in the "miracle of San Martin"

\section{Abstract}

This article studies the case of alternative development in San Martín, known by several drug enforcement agencies as the 'miracle of San Martin.' First, this article presents a theoretical framework, in which territorial control and state capacities are the main determinants of successful alternative development. With this theoretical framework, this article establishes three different periods of alternative development implementation in the San Martin region. During the first of them, from 1981 to 1989 , the rise of drug trafficking and the presence

* Licenciado en Sociología y asistente de coordinación del Grupo Interdisciplinario de Enfoques Cualitativos para el Estudio de la Política del Departamento de Ciencias Sociales de la PUCP.

+ Recibido el 15 de febrero de 2017; aceptado el 22 de mayo de 2017. 
of subversive organizations in San Martin impeded the implementation of alternative development programs. During the second period, from 1990 to 2002, the fall of coca prices, the spread of the Fusarium oxysporum fungus, the suspension of forced eradication of coca, and the weakening of the subversive organizations opened an opportunity for the implementation of alternative development. Finally, during the last period, from 2003 to 2015, the engagement of political authorities in decision-making regarding alternative development and a favorable international context towards the prices of alternative crops helped to establish the definitive model of alternative development in San Martin.

Key words: crime, agrarian economics, internal politics, economic and social development, aid for development. 


\section{Presentación de la investigación}

El denominado «milagro de San Martín» es presentado como uno de los mayores logros en materia de desarrollo alternativo del mundo por las agencias de lucha contra las drogas. Este éxito ha sido recogido en varios documentos oficiales que buscan dar cuenta del mismo y que, a la vez, se ocupan de destacar sus logros (UNODC, 2012; Devida, 2015; USAID, 2012). Entre los principales indicadores que demuestran la excepcionalidad de San Martín, se encuentra la reducción sostenida de hectáreas de hoja de coca, la cual descendió de las estimaciones de entre 28600 y 90000 hectáreas a inicios de la década de 1990 a tan solo 311 en 2014 (García, 2013a; INEI, 1994; UNODC, 2015). Este hecho es aún más sorprendente si se considera que esta reducción se mantuvo incluso en el segundo boom de la coca, es decir, durante el contexto de crecimiento sostenido de la superficie cultivada de hoja de coca que experimentó el país desde el año 2000. Como se observa en el siguiente gráfico, San Martín ha tenido un descenso sostenido en su cantidad total de hectáreas de hoja de coca, incluso durante el segundo boom de la coca.

\section{Gráfico 1. Cantidad de hectáreas de hoja de coca en San Martín y otras regiones,} 1992-2011

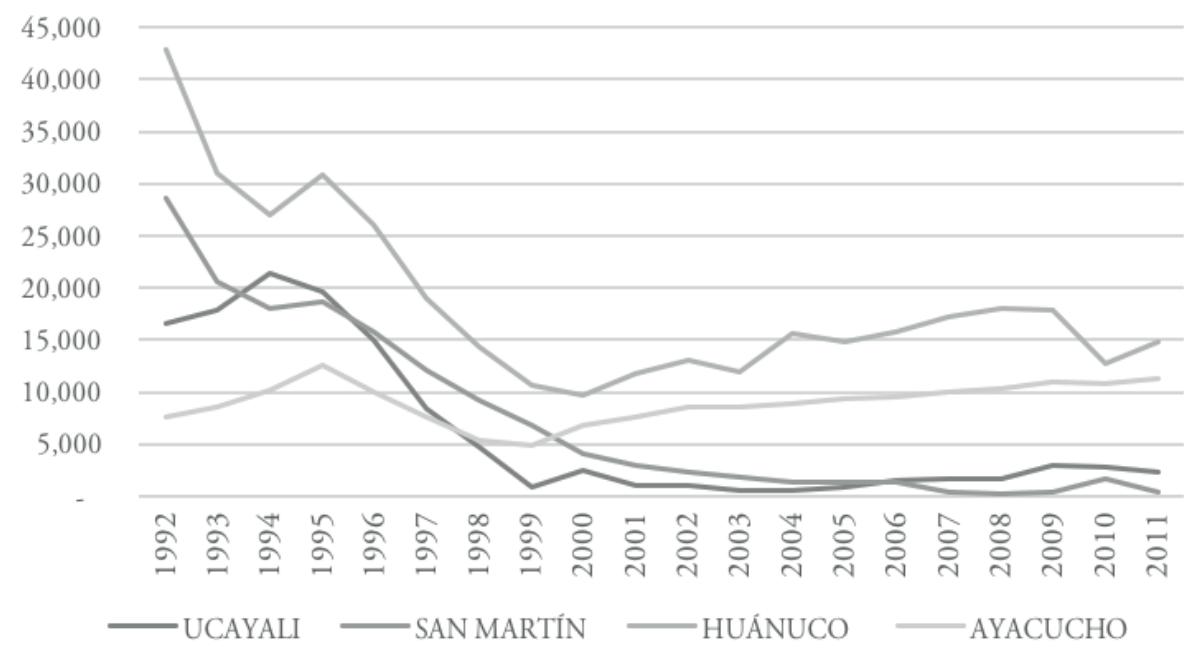

Fuente: García (2013a, 2013b, 2013c, 2013d). Elaboración propia. 
El descenso de la superficie cultivada de coca ha implicado una reducción de la importancia de este cultivo en la economía de San Martín. En primer lugar, la producción de coca destinada al narcotráfico descendió de más de 27 mil toneladas métricas en 1994 a menos de 4 mil para el año 2012. En segundo lugar, si bien la coca representaba hacia 1994 el 75,18\% del valor bruto de la producción agraria de la región — superando ampliamente a los cultivos lícitos—, hoy en día ese porcentaje se ha reducido notablemente (García, 2013a).

La región San Martín escapó al segundo boom de la coca no solo por la reducción del cultivo de coca, sino también por la sostenida expansión de cultivos lícitos. Durante la primera década de 2000, la región San Martín concentró la mayor cantidad de hectáreas apoyadas por los programas de desarrollo alternativo. Entre los años 2002 y 2010, el apoyo hacia los cultivos alternativos como el cacao, café, palma aceitera y palmito llegaron a abarcar un total de 42 604 hectáreas (SIMDEV, s/f). En una sola década, la producción de café se ha duplicado y hoy representa casi el $20 \%$ de la exportación nacional de este producto (UNODC, 2012, p. 54). El caso del cacao es aún más llamativo, pues la exportación de este producto se ha triplicado en el mismo lapso de tiempo. Sin embargo, el cultivo alternativo que ha llamado más la atención por su importancia para el consumo interno es la palma aceitera, la cual es altamente demandada para la industria de alimentos y de biocombustibles (UNODC, 2012, p. 54).

Pese a la existencia de cifras que dan cuentan del cambio sucedido en San Martín, el proceso por el cual dicha transformación se dio no ha sido todavía suficientemente estudiado. El presente artículo busca contribuir a la comprensión del desenvolvimiento de la lucha contra las drogas y de desarrollo alternativo en San Martín. En particular, se realizará un rastreo de los procesos acontecidos en el ámbito de las iniciativas estatales y de desarrollo alternativo llevadas a cabo en la región. De esta manera, este artículo busca mostrar el proceso de transformación de la región San Martín: de haber sido el principal centro del tráfico ilícito de drogas - y un importante foco subversivo- a convertirse en el «milagro» o «modelo» de desarrollo alternativo.

Este artículo se divide en cinco partes. En la segunda parte, se realizará la discusión en torno a los elementos teóricos que guían esta investigación. En la tercera parte, se presentará la metodología empleada. En la cuarta parte, se muestran los hallazgos de la investigación, los cuales están divididos en tres etapas históricas de intervención del Estado y el desarrollo alternativo. En la quinta parte, se presentan las reflexiones finales de la investigación. 


\section{Elementos Teóricos DEL ESTUdio}

El «milagro de San Martín» no debe ser entendido solamente como un caso exitoso de las políticas de control de drogas centradas en la oferta, sino también como un caso de construcción del Estado. Como política de Estado, el desarrollo alternativo es definido como «un proceso destinado a reducir y eliminar el cultivo de plantas que contengan estupefacientes, así como sustancias sicotrópicas, mediante la adopción de medidas de desarrollo rural expresamente concebidas con tal fin» (Devida, 2015, p. 23). Sin embargo, este proceso encuentra una serie de dificultades para llevarse a cabo. Si se toma en consideración que las plantaciones de cultivo ilícitos como la hoja de coca se realizan en zonas donde tanto la presencia como la legalidad del Estado no tienen lugar o, en todo caso, son bastante limitadas - las denominadas zonas marrones (O’Donnell, 1993)_, es posible comprender parte de los obstáculos que enfrenta el Estado para construir la legalidad en estos espacios.

El territorio no es un espacio neutro. Por el contrario, este es el producto de una lucha política. Tal como lo refleja el caso de estudio de esta investigación, existe una competencia entre actores estatales y no estatales por el control del territorio. Como se ha señalado previamente, en San Martín existía la presencia tanto de organizaciones de tráfico ilícito de drogas como de organizaciones subversivas. Sin embargo, no todos los desafiantes del Estado son iguales; por el contrario, estos varían en sus objetivos y estrategias. Como sostiene Eaton (2012, p. 648), mientras las organizaciones subversivas buscan controlar el territorio para derrotar al Estado y tomar el control del gobierno, los grupos de traficantes buscan principalmente controlar el territorio para poder asegurar sus actividades económicas ilícitas. Así, para que el Estado logre implementar exitosamente el desarrollo alternativo o cualquier política que afecte los intereses de sus desafiantes, este debe derrotarlos o desplazarlos como una precondición necesaria para el restablecimiento de su autoridad. De esta manera, una vez garantizado el control territorial y las condiciones mínimas de seguridad, puede el Estado emprender otro tipo de acciones, como la promoción del desarrollo alternativo.

La no comprensión de esta secuencialidad en las intervenciones puede ser perjudicial para los Estados. Como sostiene Felbab-Brown (2013), ciertas acciones represivas de la lucha contras las drogas, como las erradicaciones forzadas de cultivos ilícitos, pueden conllevar a resultados contraproducentes. En contextos de subversión y lucha armada la represión hacia las poblaciones más vulnerables y dependientes de los cultivos ilícitos mediante estos actos genera 
una estructura de oportunidad para las organizaciones subversivas. Dada su capacidad bélica en zonas donde el Estado tiene pocas capacidades y control sobre el territorio, estas organizaciones pueden ofrecer protección a la población dependiente de los cultivos ilícitos y a otros eslabones del tráfico de drogas. Este hecho no solo implica la generación de beneficios económicos para las organizaciones subversivas - que a su vez conllevan a un fortalecimiento de su capacidad bélica一, sino también la generación de capital político y una mayor legitimidad frente al Estado.

Las acciones del Estado denotan el tipo de relación que este mantiene con la sociedad. En este punto, es importante rescatar las teorías de Michael Mann (1984) en torno al poder despótico y el poder infraestructural del Estado. El primer tipo se caracteriza por una imposición de las políticas estatales sin negociación previa con la sociedad civil ni las élites locales, mientras que el segundo implica la penetración efectiva del Estado en la sociedad civil y las élites a través de la negociación para así implementar las decisiones políticas. En el caso de estudio de esta investigación, se aprecia efectivamente una transición en el uso del poder del Estado.

Inicialmente, el uso del poder despótico fue el predominante en el contexto del auge de la subversión y del tráfico de drogas. Sin embargo, es necesario distinguir entre dos periodos diferenciados en el ejercicio de este poder. Durante la década de 1980 se llevó a cabo una represión generalizada bajo la perspectiva de la lucha contra el narcoterrorismo, la cual sostenía que los cocaleros y los terroristas se encontraban aliados (Tarazona-Sevillano y Reuter, 1990). Es solo tras el fracaso de este tipo de accionar que el Estado optó por implementar una represión focalizada de carácter secuencial. De esta manera, el Estado enfocó sus recursos y esfuerzos en la derrota de su amenaza principal, las organizaciones subversivas, para solo luego enfrentarse al tráfico ilícito de drogas.

Una vez que el Estado logró la "pacificación» en San Martín, a mediados de la década de 1990, una serie de factores abrieron una ventana de oportunidad para el desarrollo alternativo. En esta sección no se detallarán cada uno de estos, con excepción del traslado de los cultivos ilícitos hacia otras zonas, es decir, el efecto globo. En el ámbito de la producción de drogas, el efecto globo hace referencia a la creación de nuevas zonas de producción de cultivos ilícitos ante la intervención de un área de producción ya establecida. En breve, se trata de que cuando una zona es intervenida, simplemente otra nueva zona de producción aparecerá (UNRISD, 1994). Como se verá a lo largo del texto, el efecto globo - así como otros elementos externos — ha sido un factor fundamental para el éxito de San Martín. 
Si bien los esfuerzos para implementar el desarrollo alternativo iniciaron en 1981, la falta de condiciones mínimas de seguridad, y la presencia de narcotraficantes y organizaciones subversivas impidieron su éxito. Fue solo después de que el Estado restableció el control territorial que el desarrollo alternativo pudo implementarse efectivamente. Sin embargo, para que este lograra consolidarse fue necesario que el Estado permitiera la negociación con las élites y la participación de la sociedad civil. Esto implicó una progresiva transición hacia el poder infraestructural del Estado en el caso de San Martín, el cual permitió la participación activa y propositiva de una serie de actores locales y regionales en el proceso.

\section{Metodología}

La metodología empleada consistió en la revisión bibliográfica de literatura especializada, de documentos oficiales del Estado y de organismos de la cooperación internacional. Se revisó la totalidad de monitoreos de cultivos de coca de Naciones Unidas. Se revisaron también varios informes de consultorías realizadas para estos organismos. Otras fuentes revisadas incluyen leyes, decretos ley y decretos supremos concernientes al tema del tráfico de drogas. Finalmente, estas tareas fueron complementadas con un trabajo de campo realizado en las provincias de Tocache y Mariscal Cáceres — las regiones más afectadas por el tráfico ilícito de drogas y la subversión en San Martín-, en donde se entrevistó a autoridades locales, funcionarios de las agencias antidrogas y, finalmente, a ex agricultores cocaleros y actuales participantes de los programas de desarrollo alternativo.

\section{Hallazgos del estudio}

Desde finales de la década de 1970, el Perú se ha consolidado como uno de los principales eslabones del tráfico ilícito de drogas a través de la producción de hoja de coca, pasta básica de cocaína y clorhidrato de cocaína. Ante este escenario, el Estado peruano implementó una serie de mecanismos centrados en la reducción de la oferta de drogas. Una de las principales estrategias ha sido la implementación del desarrollo alternativo. Tras varios éxitos parciales y desaciertos durante tres décadas, el Perú posee hoy uno de los casos más importantes de éxito de desarrollo alternativo a nivel mundial, el denominado «milagro de San Martín». 
La implementación del desarrollo alternativo ha sufrido varias transformaciones desde su inicial formulación. Estos cambios han respondido a la necesidad de adaptación ante el cambiante contexto en el que operaban, y también a un aspecto estratégico. Los hallazgos de la investigación permiten establecer tres periodos del desarrollo alternativo en San Martín claramente diferenciados. El primero de ellos transcurrió entre los años de 1981 a 1989, en donde el Estado fue el principal ejecutor de los programas de desarrollo alternativo en un contexto caracterizado por la situación de boom de la coca y la prevalencia de la violencia de la subversión y el narcotráfico. El segundo periodo comprende los años desde 1990 hasta 2002. En este periodo se distinguen claramente dos etapas, siendo la primera de ellas de 1990 a 1994 y la segunda de 1995 a 2002. Durante la primera etapa, el desarrollo alternativo se vio reforzado por el apoyo del gobierno de Fujimori y se erigió como la vía para mantener la pacificación en las zonas anteriormente controladas por la subversión. Sin embargo, fue recién en la segunda mitad de la década de 1990 que los programas de desarrollo alternativo se empezaron a ejecutar en gran escala. Por último, se abordará el periodo que comprende desde 2003 hasta 2015, en el que la participación de las autoridades políticas en las decisiones del desarrollo alternativo y el contexto internacional favorable de los precios de los cultivos alternativos ayudaron a sentar las bases del «modelo definitivo» de desarrollo alternativo de San Martín.

\subsection{Persistiendo en el fracaso: limitantes territoriales para el despliegue del desarrollo alternativo (1981-1989)}

La primera etapa de implementación del desarrollo alternativo en el país (1981-1989) estuvo caracterizada por la situación de inseguridad debido al predominio de Sendero Luminoso, el MRTA y los traficantes de drogas internacionales y locales en la región San Martín, especialmente en la parte sur donde se encuentra el valle del Alto Huallaga. El programa de mayor dimensión en esta etapa fue el Proyecto de Desarrollo del Área del Alto Huallaga (UHAD por sus cifras en inglés), firmado el 15 de setiembre de 1981 entre el Perú y USAID. Este proyecto consistía en «desarrollar y aplicar paquetes de producción agrícola y fortalecer los servicios de apoyo del sector público paralelamente con el programa peruano de erradicación de la coca en la región del Alto Huallaga» (USAID, 1986, p. 3). Este programa incluía al Programa Especial Alto Huallaga (PEAH), cuyo objetivo era sustituir la hoja de coca por cultivos alternativos como el café y el cacao. 
El PEAH, como la mayor parte de iniciativas productivas estatales en San Martín, encontró dos grandes dificultades que complicaron su implementación. En primer lugar, las condiciones de inseguridad impidieron que el programa pudiera llevarse a cabo con normalidad, debido a los ataques y amenazas por parte de los grupos subversivos. En segundo lugar, la sustitución de cultivos no resultaba atractiva para los agricultores, pues la coca contaba con un elevado precio durante aquellos años. Además, la red vial existente se encontraba sumamente deteriorada y obligaba a los agricultores a emprender difíciles y costosos trayectos para poder sacar su producción, lo cual desincentivaba a estos a cosechar cultivos lícitos.

El diseño del PEAH complicaba aún más esta situación, pues restringía la participación en el programa solo a los campesinos certificados por el Corah ${ }^{1}$ como no cocaleros (McClintock y Vallas, 2005, p. 197). Si un campesino deseaba participar del programa, este debía erradicar su coca a cambio de una mínima cantidad de dinero por hectárea. Sin embargo, debido a que el Corah tenía un ritmo más acelerado que el $\mathrm{PEAH}$, los campesinos participantes percibieron que su coca era erradicada sin sustitución ni compensación alguna, lo cual aumentó su desconfianza hacia el desarrollo alternativo (Obando, 1993, p. 83).

La situación de inseguridad aumentó a tal extremo que varios funcionarios dentro del área del proyecto UHAD abandonaron la zona en la segunda mitad de la década de 1980, debido a la creciente inseguridad vinculada al narcotráfico y los dos grupos terroristas (TRD, 1992, p. 8). Con esta disminuida presencia estatal, los proyectos de desarrollo alternativo se vieron aún más vulnerables. Así, en 1985, durante los trabajos de titulación de tierras, el PEAH fue atacado y tres topógrafos fueron asesinados (TRD, 1992, p. 11). Debido a esto, las actividades de investigación sobre los suelos y los cultivos adecuados para sustituir a la coca fueron suspendidas, y a partir de 1986 los trabajadores del PEAH debieron ir acompańados por la Guardia Republicana, y poco después se vieron obligados a permanecer recluidos en su sede de Aucayacu.

En vista de la situación crítica del programa, en 1986 se realizó la «Segunda enmienda» del UHAD, en cuyo documento se evaluó el proyecto. El documento expresa la profunda insatisfacción de considerar que el PEAH era prácticamente la única entidad del Gobierno peruano en el valle y sostenía que no se podía trabajar en una «tierra de nadie». Efectivamente, según un documento de la General Accounting Office, el PEAH fue la única entidad gubernamental

\footnotetext{
El Proyecto Especial de Control y Reducción de Cultivos Ilegales en el Alto Huallaga, conocido como el Corah, es la organización encargada de la erradicación manual de la hoja de coca.
} 
presente en el Alto Huallaga durante el mayor periodo de violencia (GAO, 2002, p. 33). Si bien esta información es difícil de ser verificada, las evaluaciones de los programas y los documentos sobre el desarrollo alternativo en la época apuntan a una situación de ingobernabilidad en la parte sur de San Martín, correspondiente al valle del Alto Huallaga. En efecto, el temor a las represalias de Sendero Luminoso hizo que en el área del proyecto UHAD no se presentaran candidatos para las elecciones municipales de 1989; con excepción de Uchiza, donde participó el Frente Solidaridad Uchizana (Manrique, 2016, pp. 127-128).

En cuanto a las propuestas para mejorar el proyecto, la «Segunda enmienda» planteaba la necesidad de trasladar el énfasis de los servicios de agricultura hacia la provisión de una serie de servicios de desarrollo para la comunidad. El mantenimiento de carreteras pasó a ser el primer rubro de inversión y los servicios de desarrollo para la comunidad (agua potable y saneamiento ambiental) cobraron un papel más importante en el proyecto. La segunda enmienda del proyecto también contemplaba la extensión del periodo del proyecto y la expansión de las comunidades beneficiarias del mismo.

Sin embargo, estos esfuerzos no alteraron de ninguna manera la expansión de la coca. Aun pese al inicio de otros proyectos de desarrollo rural del Programa de las Naciones Unidas para la Fiscalización (PNUFID —ahora UNODC-) en 1986 para producir y comercializar los cultivos alternativos sin exigir la autoerradicación de la coca como precondición para participar (Parra, 2014), las hectáreas de coca siguieron incrementándose en el país y llegaron a las 121300 ha en 1990 (UNODC, 2004, p. 241). Es más, según estadísticas del Estado peruano, en 1990 la extensión de coca en San Martín llegó hasta las 90000 hectáreas (INEI, 1994). En este contexto, poco habría importado si el encargado de ejecutar estos proyectos hubiera sido el sector privado o alguna ONG, pues no existía ningún sustituto con una rentabilidad cercana a la coca. Además, la escasa presencia territorial y las condiciones de inseguridad generadas por la subversión y el narcotráfico dificultaron no solo el éxito del desarrollo alternativo, sino incluso su propia existencia. Las erradicaciones llevadas a cabo por el Corah perjudicaron al Estado en su lucha política y militar contra Sendero Luminoso y el MRTA, los cuales se vieron fortalecidos al vincularse con el incesante negocio del narcotráfico y generaron una situación aún más adversa para implementar el desarrollo alternativo.

Esta situación se volvió insostenible cuando Sendero Luminoso atacó el puesto policial de Uchiza (Base DOES-6-Uchiza). En este ataque, fueron asesinados cuatro civiles y diez policías, además catorce miembros de esta 
institución resultaron heridos. Este suceso rápidamente se convirtió en un escándalo nacional que obligó a reconsiderar las estrategias estatales implementadas. Poco después, el general Alberto Arciniega asumió la dirección del Frente Huallaga y suspendió las erradicaciones de coca, pues consideraba que estas acciones fortalecían a Sendero. Además, con el objetivo de recobrar el control estatal sobre el territorio, se establecieron varias bases militares en Uchiza, Tocache, Santa Lucía, Madrea Mía, Nuevo Progreso, Pizana, Tulumayo, Punta Arenas, entre otros (CVR, 2003).

Con el respaldo de la población, la cual había sido organizada autónomamente en rondas campesinas (Espinosa, 1995, p. 59) y por los militares en Comités de Autodefensa (Durand, 2014, p. 160), el Ejército pudo infringir fuertes bajas a Sendero y el MRTA. Sin la protección de estos, los traficantes tuvieron que desarrollar sus actividades en una arena cada vez más hostil, donde además enfrentaban una rotunda caída de precios, un mayor control territorial del Estado y la difusión del hongo Fusarium oxysporum, conocido localmente como la «seca-seca», debido a que secaba los cultivos y no les permitía volver a crecer. El origen de este hongo es incierto, pero no sus efectos. Entre 1991 y 1993, este hongo devastó al menos un 30\% de las plantaciones de coca en el valle del Alto Huallaga (McClintock y Vallas, 2005, p. 219) y al menos un 40\% de los cultivos de coca entre Tingo María y Tocache (PNUFID, 1995, p. 9). Es en este favorable contexto que el recientemente electo presidente Alberto Fujimori tomó una nueva dirección para implementar el desarrollo alternativo.

\subsection{De las palabras a los hechos: la lenta transición hacia el desarrollo alternativo en San Martín y el compromiso peruano con la lucha contra las drogas (1990-2002)}

El segundo periodo de implementación del desarrollo alternativo en el país (1990-2002) puede dividirse en dos etapas. La primera corresponde a los años desde 1990 hasta 1994. Durante estos años se superpusieron una serie de sucesos inusitados en la región: la difusión del hongo Fusarium oxysporum (Rojas, 2005), el «efecto globo» interno y externo, los cuales conllevaron a una rápida caída de precios de la coca. Estos hechos afectaron notablemente a los campesinos, quienes al percatarse tempranamente de la caída de los precios de la coca buscaron nuevas fuentes de ingreso para no caer en la pobreza. La pérdida de la rentabilidad de la coca y la derrota-retirada de la subversión y el narcotráfico fueron acompańadas por la creciente presencia del Estado en la región y el restablecimiento de las condiciones de seguridad. Fue recién durante 
la segunda etapa de este periodo (1995-2002) que la creciente presencia del Estado y las mayores condiciones de seguridad facilitaron la ejecución de los programas de desarrollo alternativo.

\subsubsection{La doctrina Fujimori y el desarrollo alternativo a inicios de la década de 1990 (1990-1995)}

A diferencia de sus predecesores, Alberto Fujimori cuestionó la política de drogas vigente en el país. Desde su perspectiva, el carácter represivo de esta no había hecho más que fortalecer a la subversión. De esta manera, en base al éxito de Arciniega en el Alto Huallaga, Fujimori suspendió las erradicaciones de coca en el país. En 1990 Fujimori presentó la «Doctrina Fujimori sobre política de control de drogas y desarrollo alternativo». Esta estrategia buscaba integrar a los cocaleros a la economía formal a través de la provisión de títulos de propiedad y de créditos para sustituir los cultivos ilícitos (Rojas, 2005, p. 192). En el documento, Fujimori señalaba que los cocaleros eran informales y que era su falta de acceso a las instituciones legales la que les impedía articularse con la economía de mercado. Por ello, proponía «crear las condiciones para el desarrollo mediante una real economía de mercado, en todo el Perú, y en particular en la zona de cultivo de la hoja de coca» (Fujimori, 1990, p. 2).

Siguiendo con lo establecido en la doctrina Fujimori, a fines de 1990, se creó la Autoridad Autónoma para el Desarrollo Alternativo (Cotler, 1999, p. 202). Sin embargo, pese a la importancia que Fujimori le concedió inicialmente al desarrollo alternativo, esta institución nunca entró en funcionamiento. En diciembre de 1991 se aprobó el decreto legislativo 753, Ley de Bases de la Estrategia Integral de Desarrollo Alternativo para Erradicar el Tráfico Ilícito de Drogas con la Participación de la Población, con el cual se reafirmó el papel de las organizaciones de cocaleros como «interlocutores válidos». Este decreto legislativo planteaba la creación del Instituto de Desarrollo Alternativo (IDA) con la finalidad de conducir el desarrollo alternativo con la participación de la población (Parra, 2014).

El golpe de Estado de Fujimori el 5 de abril de 1992 puso en suspenso el apoyo estadounidense al Perú en la lucha contra las drogas. Si bien el Gobierno peruano tuvo tensiones con Estados Unidos al no desear retomar las erradicaciones de coca, sí estaba dispuesto a cooperar en la interdicción. Con ese fin, el Perú renovó su cooperación con Colombia y emprendió una represión policial, en donde participaron el SIN, la DEA y el Gobierno colombiano (Cotler, 1999, p. 237). Estas acciones represivas de la lucha contra las drogas satisficieron al gobierno de los Estados Unidos. 
En vista de que era necesario contemplar el tema del desarrollo alternativo e institucionalizar la lucha antidrogas para obtener la ayuda estadounidense, en ese mismo año el Gobierno peruano promulgó el «Plan Nacional de Prevención y Control de Drogas 1994-2000». Con este documento el Perú se comprometió a otorgar la más alta prioridad al desarrollo alternativo a la par que incidía en la necesidad de fortalecer al Estado en las zonas productoras de coca (Cotler, 1999 , p. 235). En vista de los avances realizados por el Gobierno peruano en su compromiso con la lucha contra las drogas y en la captura de importantes narcotraficantes gracias a la reorganización de la policía antidroga, USAID ofreció otorgar, a partir de 1995, treinta millones de dólares para impulsar el desarrollo alternativo. Debido a las mismas razones, las Naciones Unidas renovaron su colaboración para invertir en proyectos de desarrollo alternativo. Sin embargo, más allá de los múltiples compromisos y acuerdos ratificados por el Gobierno peruano en materia de lucha contra las drogas, ¡cuál fue el desempeño de los programas de desarrollo alternativo implementados durante la primera mitad de la década de 1990 ?

Como ya se mencionó, el desarrollo alternativo tuvo una oportunidad inédita a inicios de esta década, pues por primera vez se había logrado el sueño de los estrategas antinarcóticos: lograr que los productos alternativos tengan precios competitivos al de la coca. La expansión del hongo Fusarium oxysporum y el debilitamiento de Sendero Luminoso y el MRTA golpearon fuertemente las porciones de San Martín y Huánuco en el valle del Alto Huallaga — donde se ubicaban la mayor cantidad de cultivos - donde se producía coca. Sin la protección armada y con un Estado más presente, se generó el «efecto globo» dentro del tráfico de drogas tanto a nivel interno — subnacional- como externo — internacional- Dentro de la misma región San Martín, los cultivos se trasladaron desde el Alto Huallaga hacia el Huallaga Central y el Bajo Huallaga, es decir hacia el centro y norte de la región. Sin embargo, la mayor parte de los cultivos fueron trasladados hacia otras regiones con una presencia estatal aún más baja, como Aguaytía o Apurímac (Roncken, 1999). Esto no solo implicó un importante movimiento de los cultivos, sino también un gran flujo de personas. Los siguientes testimonios muestran parte de este proceso.

Los narcotraficantes se iban a otras zonas a comprar porque al no producir en esta zona, sembraban en otros lugares como el caso del VRAE o Palcazu [...] y eso ha hecho de que la gente empiece a hacer un éxodo masivo de acá a otras zonas y los pocos que han quedado empezaron a sobrevivir de lo que ya podían cultivar: la yuca, la papaya, el plátano (Cecil del Águila, exagricultor y profesor del Instituto Superior Tecnológico Alto Huallaga). 
Llegó un momento donde la coca que había quedado poco que no tenía ya ni precio, quién te lo iba a comprar. [Hubo] inmigración ${ }^{2}$, o sea, salir del lugar, un éxodo total del pueblo y ahí nomás se consolidó la erradicación total con la fumigación y ahí quedó en nada (Alfonso Aguire, exagricultor cocalero y presidente de la Asociación Central de Palmicultores de Nuevo Paraíso).

En cuanto al efecto globo internacional, la constante presión del Estado y las dificultades para el tráfico ilícito de drogas en las regiones del valle del Alto Huallaga motivaron que los traficantes colombianos optaran por cultivar la hoja de coca en su país en un contexto de reestructuración de su industria de la cocaína (McClintock y Vallas, 2005, p. 220). La tendencia del efecto globo externo se observa en el siguiente gráfico.

\section{Gráfico 2. Cantidad de hectáreas de hoja de coca en Perú y Colombia, 1991-2000}

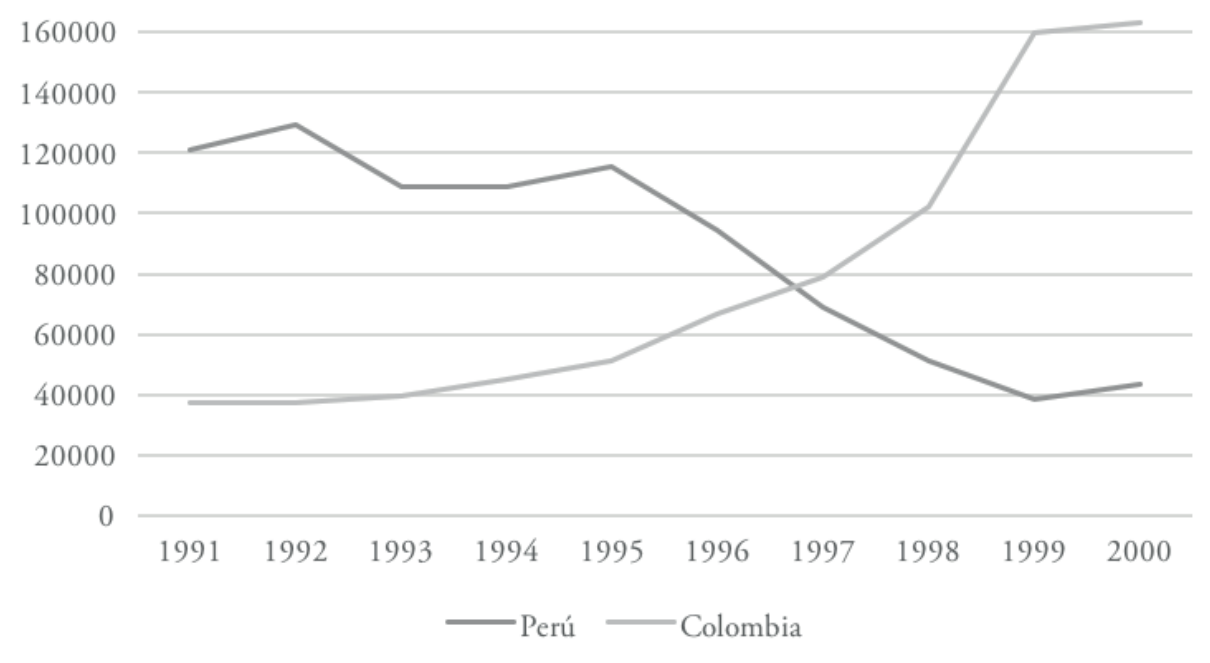

Fuente: Manrique (2015).

Los sucesos descritos líneas arriba desestabilizaron la economía del narcotráfico. Tanto la coca como la pasta básica de cocaína se vieron seriamente afectadas. La primera cayó de más de \$4 en 1994 a menos de \$1 — precio que se mantuvo de esta forma por varios años- (Kay, 1999, p. 108), mientras que la segunda cayó de $\$ 850$ a $\$ 100$ tan solo entre enero y setiembre de 1995 (Thoumi. 2003, p. 135).

\footnotetext{
2 Quiere decir emigración.
} 
El caos generado por esta caída de precios hizo que la población considerara por fin algún interés en transitar hacia las actividades lícitas. USAID aprovechó esta situación e implementó una tercera fase para el proyecto UHAD para los ańos 1992-1993. En esta etapa, USAID incorporó tanto el principal elemento de la primera fase de intervención, la sustitución de cultivos, como el de la segunda etapa, el desarrollo de las comunidades. Asimismo, subrayó que el principal objetivo de esta nueva etapa era:

[...] apoyar a los objetivos de desarrollo alternativo del gobierno peruano en el valle del Huallaga a través del fortalecimiento de los gobiernos locales y de la participación de las comunidades en el proceso de desarrollo alternativo, mejorar la infraestructura física y social y promover actividades agrícolas que reemplacen los cultivos ilícitos (Acción Agraria, 1994, p. 3).

Bajo esta nueva visión de promoción del desarrollo alternativo, el programa incluyó el valle del Huallaga Central, en donde los cultivos de coca habían crecido levemente a causa del efecto globo. Dentro de los programas de desarrollo alternativo, USAID comenzó a apoyar cultivos de impacto rápido para suplir las carencias ocasionadas por el vacío de la coca a través de plátano (Tocache y Biabo), algodón (Ponaza, Biabo y Juanjuí), maíz (Ponaza), papaya (Tocache), palmito (Tocache y Uchiza) y naranjas (Juanjuí).

Con respecto al trabajo realizado por Naciones Unidas en San Martín específicamente el PNUFID_- es necesario señalar que desde un inicio este tuvo una localización geográfica más amplia y una estrategia de intervención que favorecía la formación y fortalecimiento de las organizaciones de agricultores. Desde 1993, Naciones Unidas incorporó el enfoque de género para llevar a cabo sus proyectos. Los resultados permitieron que las mujeres campesinas se conviertan en beneficiarias de estos programas y que puedan tener acceso a mayores recursos económicos. Poco más de un año después, más de dos mil mujeres se habían organizado en cinco asociaciones regionales. Fue de esta manera que, a través de la participación voluntaria de la población, los programas de desarrollo alternativo de USAID y Naciones Unidas poco a poco se fueron abriendo paso en el nuevo contexto que se había iniciado tras la caída de la coca.

\subsubsection{Institucionalización de la politica de control de drogas y auge de los pro- gramas de desarrollo alternativo (1995-2002)}

En vista de la posibilidad de establecer una economía sin drogas al menos en algunas zonas del país, en 1995 los gobiernos del Perú y de los Estados Unidos firmaron el Proyecto de Desarrollo Alternativo (PDA) para implementarlo en 
un periodo de cinco años (USAID, 1995, p. 1). En términos comparativos, el PDA era mucho más grande y ambicioso que el anterior proyecto UHAD, pues tenía el objetivo de asegurar las oportunidades en las actividades económicas lícitas, promover el acceso a servicios sociales básicos, promover la participación pública en la toma de decisiones y tratar los problemas relacionados con el uso y producción de drogas en cinco valles cocaleros del país (GAO, 2002, p. 34). Estos eran en específico el Huallaga Central, Alto Huallaga (ambos en San Martín), Aguaytía, Selva Central y el valle del río Apurímac.

Con la firma de este documento los esfuerzos del Gobierno peruano para implementar el desarrollo alternativo se intensificaron. Si bien, hasta ese entonces, el Perú era reconocido como el país que más había invertido anualmente en el desarrollo alternativo en todo el mundo (UNODC, 2001), era necesario integrar adecuadamente esta estrategia con las otras acciones llevadas a cabo por el Estado en materia de control de drogas para obtener mejores resultados. Para ello, en 1996, se creó la Comisión de Lucha Contra el Consumo de Drogas (Contradrogas) con el objetivo de coordinar las acciones destinadas a sustituir los cultivos de coca, prevenir el consumo de drogas, y constituirse en el interlocutor oficial de gobiernos extranjeros y organismos internacionales (Cotler, 1999, p. 240).

Paralelamente a estos sucesos, la caída de precios de la coca había dado lugar a una reducción de las hectáreas de este cultivo. Si bien hacia inicios de la década de 1990 la cantidad de hectáreas de hoja de coca había comenzado a reducirse, a partir de la segunda mitad de la década la reducción fue de manera más acelerada (Zevallos, 2012, p. 37). Esto se debió al éxito de la interdicción aérea y al reinicio de las erradicaciones forzadas. Efectivamente, en 1996 se volvieron a ejecutar las erradicaciones forzadas en todo el país, con un total de 1259 ha erradicadas ese año, 3462 en 1997 y 7834 en 1998 (UNODC, 2010). Solo entre 1996 y 1998, se erradicaron alrededor de 2300 hectáreas de hoja de coca en la región San Martín (García, 2013a).

Pese al retorno de las acciones represivas de control de drogas, no se realizaron grandes movilizaciones en defensa de los cultivos de coca. La ausencia de esta respuesta se debió a que en ese entonces los agricultores habían dejado la coca por su bajo precio y muchos de ellos estaban cultivando otros productos con el apoyo de los programas de desarrollo alternativo o sin ellos. Además, en los territorios del Huallaga aún permanecían varias bases militares y contrasubversivas, las cuales limitaban la posibilidad de respuesta de los cocaleros.

Esta ausencia de movilización contra las erradicaciones permitió que los programas de desarrollo alternativo se extendieran sin mayores problemas en 
la mayor parte de territorios de la región San Martín. Paralelamente a los progresos del PDA, el programa de desarrollo alternativo de las Naciones Unidas comenzó un proyecto de palma aceitera a través de la capacitación a los antiguos productores y trabajadores de Endepalma — quienes, tras la quiebra de esta empresa estatal, habían obtenido algunas hectáreas como compensación social - para mejorar su producción y competir en el mercado. Asimismo, se iniciaron acciones de reforestación y conservación de los recursos naturales. Sin embargo, lo más importante fue que se incluyeron medidas complementarias de mejora de la infraestructura económica y social que correspondían a las necesidades básicas de la población (Parra, 2014).

En esta primera etapa del PDA (1995-2002), uno de los contratistas principales de USAID para implementar el Programa de Desarrollo Alternativo fue la ONG estadounidense Winrock International (UNODC, 2012, p. 106). Su labor fue apoyada por organizaciones como el Centro de Información y Educación para la Prevención del Abuso de Drogas (Cedro), Prisma, Acción Agraria, entre otros. Como señala UNODC respecto al caso de San Martín, «la estrategia de estas ONG consistió en financiar la formación de empresas privadas que tuvieran como accionistas directamente a los productores de los cultivos (con potencial de mercado) apoyados. Estas empresas recibieron apoyo en la forma de capital de trabajo y costos operativos» (2012, p. 107). Además, Winrock buscaba la participación activa de las autoridades locales y sectoriales en conjunto con la población y los entes cooperantes. Por otro lado, la estrategia de Prisma —otro de los contratistas - consistió en incrementar la producción y productividad, mejorar la calidad de los productos, así como la gestión comercial, facilitar el acceso al crédito y, finalmente, fortalecer las instituciones de base (Maldonado, 2009, p. 11). Su trabajo en el Huallaga Central y Bajo Huallaga tuvo énfasis en Tarapoto, Lamas y Bellavista a través de cultivos como el café, cacao y algodón, mientras que en el Alto Huallaga-Huallaga Central se privilegiaron las provincias de Tocache y Juanjuí para producir cacao, frutales y menestras.

Ante un posible resurgimiento de la coca, la erradicación de hoja de coca volvió a convertirse en una prioridad para el Estado. Solo entre 1998 y 2000 se erradicaron alrededor de 30 mil ha en el país (UNODC, 2010), de las cuales más de 12 mil ha pertenecían a San Martín (García 2013a). La magnitud de la erradicación generó las primeras respuestas violentas de los cocaleros, quienes comenzaron a organizarse para la defensa del cultivo de coca.

Tras el fin del régimen de Fujimori, el retorno a la democracia no conllevó a una pacificación de las cuencas cocaleras. En efecto, los reclamos en defensa de la coca se habían vuelto cada vez más violentos y esto ponía en peligro los 
programas de desarrollo alternativo. Hacia inicios de la década de 2000, la coca recuperó su precio, lo cual motivó a que miles de agricultores volvieran a dedicarse a su cultivo (UNODC, 2015, p. 70). En este contexto, el movimiento cocalero se posicionó como su principal defensor. Este retorno a la coca tuvo como consecuencia un segundo boom de la coca, que se manifestó en el incremento de las hectáreas dedicadas a este cultivo (UNODC, 2010).

En este contexto de movilización y tensión social, CARE asumió en noviembre de 2000 las operaciones del PDA por un periodo de tres años. Al igual que las demás organizaciones, CARE no solo implementó sus programas en San Martín, sino también en otras zonas cocaleras del país. No obstante, San Martín se distinguió de las demás por su menor grado de conflictividad. Uno de los factores que explican que las erradicaciones efectuadas en la segunda mitad de la década de 1990 no encontraran gran resistencia en San Martín, fue que estas fueron realizadas en un periodo de caída de precios de la coca. Otra razón se encuentra en el hecho de que la cantidad de hectáreas de coca existente era bastante menor en comparación con otras regiones. A manera de ejemplo, si se comparan las 2896 hectáreas de coca de San Martín en el año 2001 con las 11725 de Huánuco, las 17760 de Cusco o las 7560 de Ayacucho, queda claro que el problema de la coca en San Martín - y la dependencia a este cultivo - era bastante menor, y que por ende el desarrollo alternativo encontraría menos obstáculos.

Efectivamente, mientras en varias regiones del país se estaba experimentando un segundo boom de la coca, San Martín reducía progresivamente sus hectáreas de coca. Por ello, pese a contar con algunas importantes zonas cocaleras en la provincia de Tocache, como Sión o el valle de Santa Rosa de Mishollo, la región no tuvo una participación similar a la de regiones como Huánuco o Ayacucho en la lucha por la defensa de la coca. Durante la celebración de los comicios electorales del año 2002, el movimiento cocalero presentó a varios candidatos para los municipios locales de la región San Martín. Sin embargo, dado que la región había comenzado lentamente a dejar la economía de la coca desde hacía algunos años, los resultados no les fueron favorables. Pese a contar con Nancy Obregón —una de las máximas dirigentes del movimiento cocalero a nivel nacional — como candidata a la alcaldía de Tocache —el último bastión de la coca en la región-, solo un 14,9\% de los electores votaron por ella. En aquel proceso electoral el movimiento cocalero solo obtuvo una alcaldía distrital en San Martín.

El bajo apoyo hacia el movimiento cocalero se explica por el hecho de que los agricultores ya habían comenzado a ver las «bondades» de los cultivos alternativos y sentían que el movimiento cocalero no les ofrecía ninguna opción 
además del rechazo rotundo al desarrollo alternativo y las erradicaciones forzadas. Asimismo, es necesario resaltar que para varios excocaleros los cultivos alternativos les permitían contar con un ingreso seguro y no les generaba ningún tipo de riesgo de ser intervenidos por la policía (Ugarte, 2014). Los agricultores no deseaban volver a sembrar la coca debido a que conocían los problemas a los que conllevaba la ilegalidad y estaban presenciándolos nuevamente en los enfrentamientos entre el movimiento cocalero y el Estado, y en el resurgimiento de Sendero Luminoso. Los siguientes testimonios reflejan esta nueva situación en San Martín:

Se comienza a superar esta creencia que tenía doña $\mathrm{Nancy}^{3}$ con muestras de plantaciones que comienzan a dar fruto el cacao y la palma. Entonces, al ver que uno recibía $\$ 200$ por tonelada y lícito, ya comenzaron a darle la espalda. Y otro es cuando dońa Nancy se lanza a la política y llega al Congreso y ahí abandonó a toda la provincia (Francisco Pino Morillo, 56 años, exagricultor cocalero y socio directivo de la Cooperativa Agroindustrial de Tocache).

[Lo que sucedió fue que] la zona ya estaba dividida en dos facciones. Un grupo que ya estaba llevando el cultivo alternativo y había el otro grupo que rechazaba. El grupo que ya aceptó el desarrollo del cultivo alternativo ya comenzaron a ver las bondades del cacao y de la palma y había este grupo que no aceptaba y tampoco escuchaba, no permitía que tú le expliques, no quería escuchar (Néstor Sánchez Falcón, 62 años, Director de OLPESA, Tocache).

Esta situación particular de San Martín hizo que el PDA se concentre principalmente en dicha región. Hacia inicios de los años 2000, alrededor del $60 \%$ de las localidades/distritos participantes del PDA a nivel nacional tuvieron lugar en San Martín (USAID, 2002b). En este contexto favorable, en el año 2002 se dio inicio a la segunda fase del PDA: «Reducción sostenible de Cultivos Ilícitos de coca a través del Desarrollo Alternativo en áreas claves». Durante esta fase se reforzó aún más el énfasis en la región San Martín, debido a las mayores condiciones de seguridad de la región. Debido al auge de la movilización cocalera en el país, Chemonics - la nueva operadora contratada por USAID para implementar el PDA - se enfocó inicialmente solo en San Martín, y en una provincia (Leoncio Prado) y un distrito (Cholón) de Huánuco (Maldonado, 2009, p. 16). Los proyectos llevados a cabo en el marco del PDA en San Martín no solo ayudaron a promover el desarrollo en San Martín a través de los cultivos alternativos, sino también a incrementar la presencia estatal en áreas como educación y salud. En total, entre los años 1995 a 2002 el PDA había apoyado 365 obras en el sector educación y 40 obras en salud (USAID, 2003, p. 7).

3 Se refiere a la opositora del desarrollo alternativo y dirigente cocalera de Tocache, Nancy Obregón. 
Las mayores condiciones de seguridad determinaron que las erradicaciones de coca se centraran en San Martín. Tan solo en 2002, el 71\% del total de erradicaciones en el país se llevaron a cabo en esta región. Muchas de estas erradicaciones se realizaron en campos de coca que habían sido abandonados, por lo que no encontraron mayores resistencias. Por ejemplo, de las más de 4500 hectáreas erradicadas en el Huallaga Central/Bajo Huallaga, casi el 80\% fueron llevadas a cabo en campos abandonados (USAID, 2002a, p. 2). Sin embargo, las erradicaciones en el sur de la región, específicamente en la cuenca del Alto Huallaga, en el área correspondiente a la provincia de Tocache, sí causaron una fuerte movilización de los cocaleros. En el año 2003, varios cocaleros realizaron tomas de carreteras e incluso intentaron tomar por asalto el municipio de esta provincia. Debido a este tipo medidas de fuerza, algunas de las intervenciones del PDA debieron interrumpirse (USAID, 2005, p. 9).

$\mathrm{Si}$ bien estas acciones pusieron en riesgo los avances logrados del PDA en la zona, estos no se detuvieron. Por el contrario, este tomó un nuevo rumbo, que fue fundamental para la posterior consolidación del desarrollo alternativo en la región. Sin embargo, es necesario destacar que este cambio no partió de una iniciativa del gobierno central o de la cooperación internacional, sino de los propios actores locales. Tras el fallido intento de toma del municipio, el alcalde de Tocache, Pedro Bogarín, decidió coordinar con entidades como Devida, USAID, Amresam y las otras nueve municipalidades provinciales de la región la formulación de una nueva estrategia para implementar el desarrollo alternativo, que sería conocida como el «Acuerdo Común».

\subsection{El «Acuerdo Común» y la consolidación del «milagro de San Martín» (2003-2015)}

El 15 de octubre de 2003 se llevó a cabo en la Plaza de Armas de Tocache la firma del «Acuerdo Común». En base a varios acuerdos anteriores con respecto a la lucha contra el tráfico ilícito de drogas y el fortalecimiento de la democracia, participación y concertación, el Gobierno Regional de San Martín, los diez Gobiernos provinciales, Devida y USAID se comprometieron con este acuerdo a respaldar los esfuerzos conjuntos para "promover actividades que contribuyan al desarrollo de una economía lícita y a eliminar el cultivo de coca con fines ilícitos en forma gradual y concertada ${ }^{4}$ mediante acuerdos con

El énfasis es mío. 
las comunidades que acepten firmar los convenios de auto-erradicación»". Con la firma del Acuerdo Común, era la primera vez en el país que un convenio de esta magnitud para establecer los programas de autoerradicación concertada era ratificado por un Gobierno regional y todos los Gobiernos provinciales.

Pese al rechazo inicial de la población cocalera, el acuerdo pronto comenzó a dar resultados. Al firmar el convenio marco, las comunidades acordaban erradicar toda o la mayoría de su coca a cambio de asistencia para el desarrollo (USAID, 2006a, p. 11). Los agricultores cocaleros prefirieron firmar el documento en lugar de correr el riesgo de que su coca fuera erradicada por el Corah sin recibir ninguna compensación. El primer caserío en firmar el "Acuerdo Común» fue el de Las Palmeras, ubicado en el distrito de Progreso, provincia de Tocache. Debido a ser el primer caserío en firmar el acuerdo común, este fue uno de los que recibió la mayor cantidad de beneficios. Pronto, al ver los beneficios de su participación, varios caseríos adyacentes empezaron a sumarse a estos proyectos. En el año 2003, además de Tocache, varios caseríos y distritos de Juanjui y de zonas cercanas a Tarapoto se sumaron al PDA. Durante los dos años siguientes, el PDA se expandió sobre todo en la zona noroeriental (Lamas, San Martín, El Dorado), central (Mariscal Cáceres) y sur (Tocache) de la región.

Un actor clave para la implementación del desarrollo alternativo fue la Asociación de Municipalidades de la Región San Martín (Amresam), la cual también participó activamente como ejecutor del PDA. A comparación de otras asociaciones de municipalidades formadas por USAID en otras regiones, Amresam destacó por la gran cantidad de proyectos que ejecutó (USAID, 2002b, p. 4). Según una evaluación realizada por USAID (2002b, p. 8), Amresam era la asociación que más había avanzado a nivel nacional en el marco del PDA en 2002, con un total de $95 \%$ de obras verificadas concluidas y con una participación de la población en la construcción del 100\% de todas las obras.

Durante estos años, otro programa que tuvo un gran impacto en la región fue el Programa de Desarrollo Alternativo Tocache-Uchiza (Prodatu). De manera similar a Naciones Unidas en la década de 1980, Prodatu no condicionaba sus programas de desarrollo alternativo a cambio de la erradicación de la coca. Este programa enfatizaba principalmente la industrialización de los productos agrícolas, la formalización de los pequeños agricultores y la necesidad de financiar sus actividades (UNODC, 2012, p. 17).

\footnotetext{
5 Documento inédito 'Acuerdo Común Entre el Gobierno Regional de San Martín, Gobiernos Locales de la Región San Martín, la Comisión Nacional para el Desarrollo y Vida sin Drogas (Devida), y la Agencia de los Estados Unidos para el Desarrollo Internacional (USAID/Perú).
} 


\section{Mapa 1. Intervenciones del PDA en San Martín}

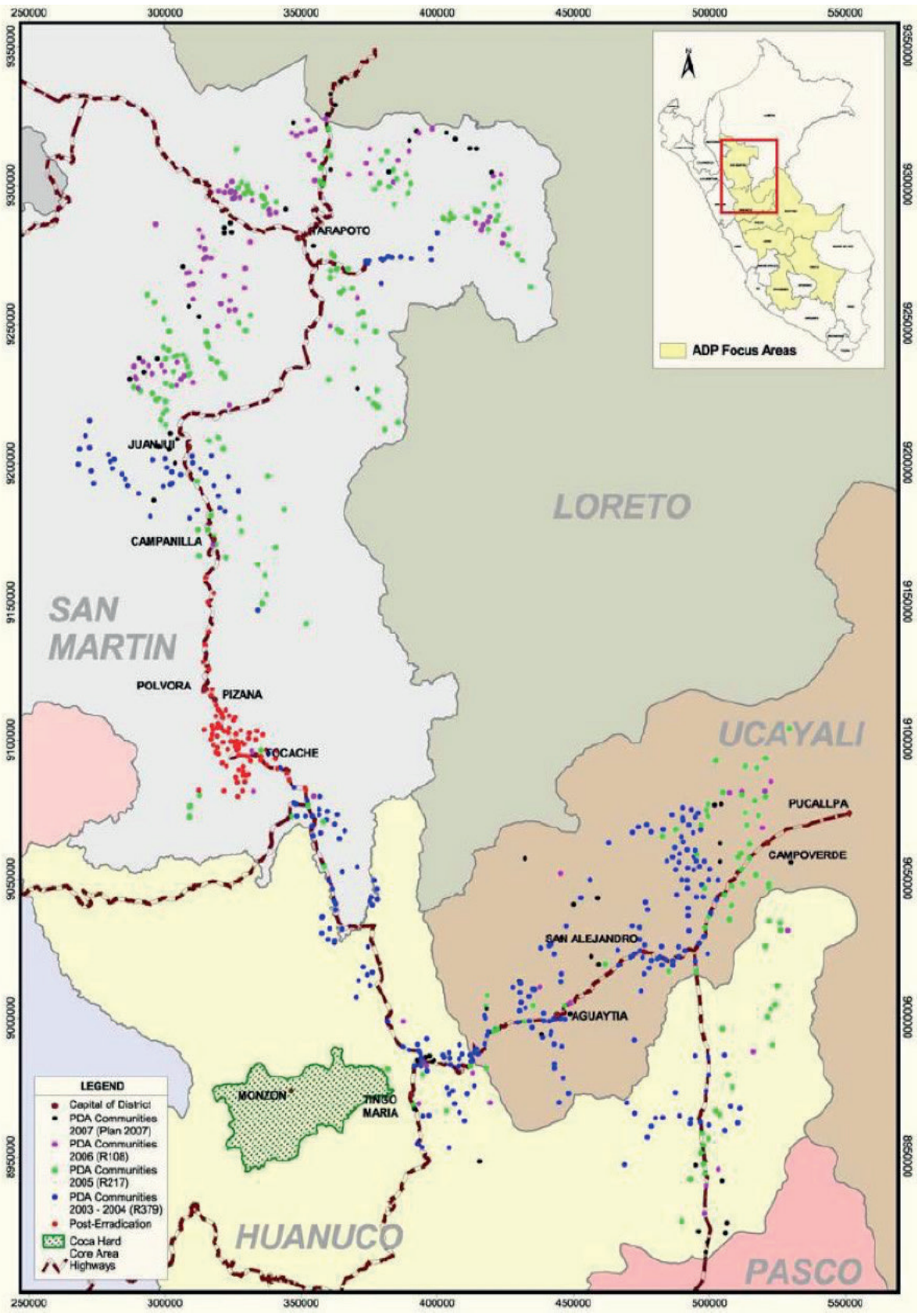

Fuente: Devida (s/f). 
Conforme los distintos programas de desarrollo alternativo fueron avanzando, la resistencia contra estos proyectos y las erradicaciones decrecieron. Bajo esta nueva situación, durante el primer lustro de la década, el total de erradicaciones, incluidas las forzadas y voluntarias en San Martín, ascendió a 14 mil hectáreas aproximadamente (García 2013a), de las cuales tan solo en la provincia de Tocache 4037 has fueron autoerradicadas voluntariamente a través del convenio marco (USAID, 2006b, p. 49). Al ańo siguiente, en 2006, el $100 \%$ de las erradicaciones anuales se llevaron a cabo en San Martín, con un total de más de 10 mil hectáreas erradicadas (García, 2013a).

En el año 2006 se produjo un hito cuando las distintas entidades de la cooperación internacional que estaban apoyando el desarrollo alternativo se alinearon para generar un solo paquete de promoción del desarrollo económico y social coherente. La elección de César Villanueva como Presidente Regional de San Martín ese mismo año, significó una importante ventaja para el desarrollo alternativo. Según Muñoz et al. (2016, pp. 48-49), las buenas relaciones de Villanueva con la cooperación internacional, la fortaleza de su partido Nueva Amazonía, y su equipo de jóvenes profesionales provenientes del sector no gubernamental, ayudaron a afianzar las bases del desarrollo alternativo en su gobierno.

El apoyo a los exportadores durante la primera gestión de Villanueva resultó fundamental para aprovechar el contexto de alza de precios de los cultivos alternativos, que se encontraban en una importante alza a nivel internacional. Esta había sido una demanda establecida por los mismos agricultores en el Plan Concertado de Desarrollo, la cual fue cumplida por el gobierno de Nueva Amazonía (Gobierno Regional de San Martín, 2010, p. 25). El siguiente gráfico muestra la situación de los precios de estos cultivos.

Como puede observarse en el gráfico 3, durante la década pasada los cultivos alternativos promocionados como el café, el cacao y la palma aceitera experimentaron una importante alza de precios. Esta situación significó una ventana de oportunidad para aquellos agricultores interesados en participar en los programas de desarrollo alternativo. Ante este contexto en particular, la coca - cuya extensión había disminuido notablemente- dejó de ser un cultivo atractivo para los agricultores en San Martín, quienes empezaron a interesarse cada vez más en los cultivos alternativos por sus altos precios y por la cantidad de programas que apoyaban su producción. 


\section{Gráfico 3. Precio de los cultivos alternativos en dólares (por tonelada)}

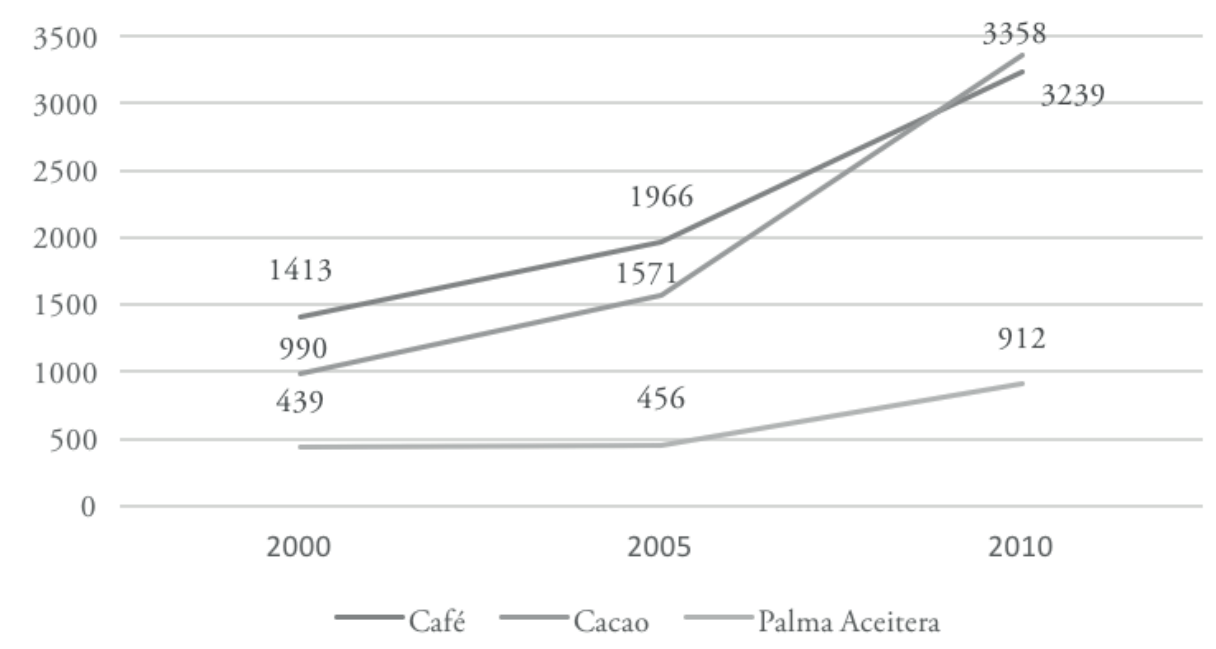

Fuente: UNODC (2012). Elaboración propia.

En 2007 inició la tercera y última fase del Programa de Desarrollo Alternativo de USAID (PDA III), la cual se prolongó hasta 2012. Durante esta etapa, se le quitó el respaldo a la erradicación concertada y se privilegió una nueva estrategia: la poserradicación y consolidación. Esta estrategia consistía en la realización de un intenso trabajo de socialización que buscaba que las comunidades afectadas por la erradicación de coca se informen y accedan a una economía lícita (Chemonics, 2012, p. 3). El modelo de poserradicación fue implementado por primera vez en Tocache entre los ańos 2006 y 2007, en donde se incluyó un extenso portafolio de opciones para los agricultores participantes como, por ejemplo, la instalación de hectáreas de cacao con semillas, sombra, para los cultivos herramientas, jornales y asistencia técnica. Esto además incluía pequeńos proyectos de infraestructura para la comunidad y el mejoramiento de carreteras terciarias.

La estrategia de consolidación estaba dirigida a hacer sostenible la producción y asegurar que los agricultores tengan las habilidades para mantener su productividad y calidad. Otro aspecto importante considerado por la estrategia de consolidación fue el fortalecimiento de las organizaciones de productores. Debido a que algunas cooperativas ya habían comenzado a resaltar por sus buenas prácticas, estas recibieron un apoyo especial a través de la estrategia de «Fortalecimiento a las grandes cooperativas». En la región San Martín, las 
principales beneficiarias fueron Acopagro (Juanjuí), Oro Verde (Lamas) y la Cooperativa Agroindustrial de Tocache (Tocache) (Chemonics, 2012, p. 15).

Cuando, en el año 2012, USAID comenzó a retirarse poco a poco de la región San Martín tras haber llevado a cabo más de 15 años de inversión en las tres fases del PDA — que suman un total de 31 ańos si se considera sus intentos iniciales a través del PEAH—, apareció otro importante proyecto en la región. En vista de los logros alcanzados por la Cooperación Alemana en San Martín a través del Prodatu y del apoyo brindado para la modernización y reforma institucional del Goresam, se dio inicio a la segunda fase del Prodatu. Este programa está diseñado para intervenir durante 5 ańos no solo en Tocache y Uchiza, sino en todos los demás distritos de la provincia de Tocache (Shunté, Pólvora y Nuevo Progreso).

En general, tanto el PDA III como el Prodatu II tuvieron como objetivo consolidar el desarrollo alternativo en la región San Martín y hacerlo sostenible en el tiempo. Los resultados mostrados por la región en materia de cultivos alternativos y disminución de hectáreas de coca sirvieron para que las organizaciones vinculadas a la lucha contra el tráfico de drogas utilicen el caso de San Martín como una demostración de que las políticas de control de drogas sí funcionan. Posteriormente, el «milagro de San Martín» fue convertido en «modelo de San Martín» (UNODC, 2012), con el objetivo de servir como un caso ejemplar de política de drogas a ser emulado en otros contextos.

\section{REFLEXIONES FINALES}

El presente artículo ha mostrado que la implementación del desarrollo alternativo ha dependido de la creciente presencia del Estado y de los proyectos de la cooperación internacional en San Martín. Como se vio, durante la década de 1980, la ausencia del control territorial del Estado y la pujante economía de la coca impidieron que estos programas se llevaran a cabo. En este sentido, la importancia de la secuencialidad de las acciones estatales (Felbab-Brown, 2013) toma relevancia. La lucha simultánea del Estado contra la subversión y el tráfico de drogas resulta contraproducente, pues favorece el establecimiento de alianzas o contratos entre ambos, a la par que el Estado pierde legitimidad al atentar contra la economía ilícita de la que dependen los campesinos. En efecto, fue solo tras la reorganización de las prioridades del Estado que este pudo tener éxito. Tras una década de fracasos, el Estado reconoció que su mayor amenaza en los valles cocaleros era la subversión. 
El objetivo que ha guiado la investigación ha sido el de mostrar cómo la progresiva construcción del Estado resultó fundamental para que el desarrollo alternativo sea exitoso. Como se ha visto, sin Estado y sin control territorial, no se puede asegurar ni la propia implementación del desarrollo alternativo. Al buscar reemplazar una economía ilícita, se afecta la reproducción de los medios de subsistencia de los agricultores cocaleros y de las organizaciones criminales y subversivas que se benefician de su industria. Por ello, es necesario considerar la importancia de la secuencialidad de las acciones del Estado en estos contextos.

Solo tras la derrota del MRTA y el desplazamiento de Sendero Luminoso, la lucha contra el tráfico ilícito de drogas recobró importancia y, debido a las mayores condiciones de seguridad, los programas de desarrollo alternativo pudieron operar libremente en San Martín. Además, de las condiciones de seguridad es necesario resaltar la oportunidad única para el desarrollo alternativo que representó la caída de precios de la coca, la devastación de la coca por el Fusarium oxysporum, y el efecto globo interno y externo.

Si bien estos cambios en las dinámicas de producción de coca, junto con el control territorial y las mayores condiciones de seguridad garantizadas por el Estado, fueron importantes para permitir que el desarrollo alternativo opere en la región, la consolidación del mismo dependió de otros factores.

Un elemento dejado de lado por los trabajos sobre San Martín (UNODC, 2012; Devida, 2015) es la participación propositiva de los actores locales. La participación y la organización social es un fenómeno antiguo en San Martín que se remonta hasta la década de 1970. Esta fue fundamental para vencer a la subversión a través de las rondas campesinas y los comités de autodefensa. Del mismo modo, esta también fue importante para proponer cambios dentro de la manera de implementar el desarrollo alternativo. La propuesta del «Acuerdo Común» surgió desde la alcaldía de Tocache y fue ratificada y firmada por todos los Gobiernos provinciales de la región. Anteriormente, el Estado había buscado combatir la coca a través del poder despótico, es decir, a través de la imposición coercitiva de sus políticas; sin embargo, la negociación y posterior aceptación, por parte del Estado central, de esta propuesta implica, por primera vez, un cambio hacia el ejercicio del poder infraestructural en materia de lucha contra las drogas en el país.

Finalmente, como se ha visto a lo largo de este artículo, la mayor parte de los sucesos señalados tomaron lugar en la parte sur de la región San Martín, principalmente en las provincias de Mariscal Cáceres y Tocache. Por ende, es complicado hablar del «milagro de San Martín» como un proceso homogéneo en toda la región. La propaganda en torno al éxito de desarrollo alternativo de la 
región estudiada ha contribuido a pasar por alto las diferencias entre los valles y provincias de San Martín. Este trabajo encontró varias limitaciones al encontrar escasa información respecto a las zonas de San Martín menos afectadas por la subversión y el narcotráfico Es necesario realizar más investigaciones en torno al narcotráfico y la subversión en las provincias y valles del centro y del nororiente de la región para poder tener una imagen más cabal del caso de San Martín.

\section{REFERENCIAS}

Cotler, Julio (1999). Drogas y política en el Perú: la conexión norteamericana. Lima: IEP.

Durand, U. (2014). The political empowerment of the cocaleros of Bolivia and Peru. Nueva York: Palgrave Macmillan.

Eaton, K. (2012). The State of the State in Latin America: Challenges, Challengers, Responses and Deficits. Revista de Ciencia Politica (Santiago), 32(3), 643-657.

https://doi.org/10.4067/s0718-090x2012000300008

Espinosa, O. (1995). Rondas campesinas y nativas en la Amazonia peruana. Lima: Centro Amazónico de Antropología y Aplicación Práctica.

Felbab-Brown, V. (2013). A State-building Approach to the Drug Trade Problem. UN Chronicle, (2), 15-19. https://doi.org/10.18356/d86c696b-en

Fujimori, A. (1990). Doctrina Fujimori sobre politica de control de drogas y desarrollo alternativo. Lima: Instituto Libertad y Democracia.

García, Jaime (2013a). La situación del narcotráfico en la región San Martín. Lima: IDEI-PUCP.

García, Jaime (2013b). La situación del narcotráfico en la región Huánuco. Lima: IDEI-PUCP.

García, Jaime (2013c). La situación del narcotráfico en la región Ucayali. Lima: IDEI-PUCP.

García, Jaime (2013d). La situación del narcotráfico en la región Ayacucho. Lima: IDEI-PUCP.

Kay, B. (1999). Violent Opportunities: The Rise and Fall of "King Coca and Shining Path". Journal of Interamerican Studies and World Affairs, 41(3), 97-127. https://doi.org/10.2307/166160

Maldonado, L. (2009). El desarrollo alternativo dentro de la estrategia antidrogas. Modelos de intervención del PDA. Manuscrito no publicado.

Mann, M. (1984). The autonomous power of the state: its origins, mechanisms and results. European Journal of Sociology, 25(2), 185-213. https://doi.org/10.1017/s0003975600004239

Manrique, H. (2015). Las bases históricas del milagro de San Martín: control territorial y estrategias estatales contra el narcotráfico y la subversión (1980-1995). Politai. Revista de Ciencia Política, 6(11), 33-51.

Manrique, H. (2016). El fin de la guerra de la cocaina: construcción del Estado y desarrollo alternativo en la región San Martín (1978-2015). Tesis de pregrado, Departamento de Sociología, PUCP, Lima, Perú.

McClintock, C. y Vallas, F, (2005). La democracia negociada: las relaciones Perú-Estados Unidos (1980-2000). Lima: IEP.

Muñoz, P., Monsalve, M., Guibert, Y., Guadalupe, C. y Torres, J. (2016). Élites regionales en el Perú en un contexto de boom fiscal: Arequipa, Cusco, Piura y San Martín (2000-2013). Lima: Universidad del Pacífico.

O’Donnell, G. (1993). Estado, democratización y ciudadanía. Nueva Sociedad, (128), 62-87.

Obando, E. (abr-jun de 1993). El narcotráfico en el Perú: una aproximación histórica. Análisis internacional, (2), 80-100. 
Obando, E. (2006). U.S. Policy toward Peru: At Odds for Twenty Years. En B. Loverman (ed.), Addicted to failure. U.S. Security Policy in Latin America and the Andean Region. Lanham, MD: Rowman \& Littlefield Publishers, Inc.

Parra, E. (2014). Desarrollo alternativo en el Perú: treinta ańos de aciertos y desaciertos. Perspectivas Rurales, (23), 85-104. Costa Rica: Universidad Nacional de Costa Rica. Disponible en: http:// www.revistas.una.ac.cr/index.php/perspectivasrurales/article/view/5599/5517

Rojas, I. (2005). Peru: Drug Control Policy, Human Rights, and Democracy. En C. Youngers y E. Rosin (eds.), Drugs and Democracy in Latin America: The Impact of U.S Policy (pp. 185-230). Boulder, CO: Lynne Rienner.

Roncken, T. (1999). "Air Bridge Denial": El éxito de un fracaso. Instituto Transacional. Recuperado de: https://www.tni.org/es/art\%C3\%ADculo/air-bridge-denial-el-exito-de-un-fracaso

Soberón, R. (1997). Corruption, Drug Trafficking and the Armed Forces. An approximation for Latin America. En M. Jelsma et al. (eds.), Crime in Uniform. Corruption and Impunity in Latin America. Disponible en: http://www.tni.org/article/corruption-drug-trafficking-and-armed-forces

Soberón, R. (2005). Narcotráfico y Derechos Humanos. En H. Cabieses, B. Cáceres, R. Rumrill y R. Soberón, Hablan los Diablos. Amazonía, coca y narcotráfico en el Perú; escritos urgentes (pp. 185-229). Quito: Abya Yala.

Tarazona-Sevillano, G. y Reuter, J. (1990). Sendero Luminoso and the threat of narcoterrorism. Washington, D.C.: CSIS.

Thoumi, F. (2003). Illicit Drugs, Economy and Society in the Andes. Baltimore, MD: John Hopkins University Press.

Ugarte, D. (2014). Después de la coca: La persistencia del cambio institucional en Tocache. Tesis de Sociología, PUCP.

Zevallos, Nicolás (2012). El impacto de las estrategias de control de cultivos en la dinámica de la defensa política de la hoja de coca en el Perú. Tesis de Ciencia Política y Gobierno, PUCP.

\section{Fuentes estadísticas e informes}

Acción Agraria (1994). Final Report. Evaluation of the Upper Huallaga Area Development Project 527-0244. Documento elaborado para USAID. Disponible en: http://pdf.usaid.gov/pdf_docs/ PDABM323.pdf

Chemonics International Inc. (2012). Transforming Communities, Transforming Lives. USAID/Peru Alternative Develpment Program III. Final Report. Disponible en: http://www.chemonics.com/ OurWork/OurProjects/Documents/Peru-PDA-III-Final-Report.pdf

CVR-Comisión de la Verdad y Reconciliación (2003). Informe final. Nueve tomos. Lima: CVR.

Devida (2015). Compendio Normativo sobre Tráfico Ilícito de Drogas y Desarrollo Alternativo. Lima: Devida.

GAO-General Accounting Office (2002). Drugs Control. Efforts to Develop Alternatives to Cultivaring Illicit Crops In Colombia Have Made Little Progress and Face Serious Obstacles. Report to Congressional Requesters. Disponible en: http://www.gao.gov/new.items/d02291.pdf

Gobierno Regional de San Martín (2010). Memoria de gestión 2007-2010. Disponible en: http:// www.regionsanmartin.gob.pe/doc_temp/memoria_gestion_2007_2010.pdf

INEI-Instituto Nacional de Estadística e Informática (1994). Perú: Compendio de Estadísticas de Producción y Consumo de Drogas 1980-93. Lima: INEI.

PNUD-Programa de las Naciones Unidas para el Desarrollo (2013). Informe sobre el Desarrollo Humano 2013. Lima: PNUD.

PNUFID-Programa de las Naciones Unidas para la Fiscalización Internacional de Drogas (1995). Mujer campesina y drogas. Situación de la mujer campesina y su actitud frente a la producción y consumo de drogas en el Huallaga, Pachitea y Ucayali. Lima: PNUFID. 
SIMDEV-Sistema de Información y Monitoreo de Devida (s/f). Mapa de cultivos alternativos apoyados por el PDA. Disponible En: http://www2.simdev.gob.pe/sig_auxiliar/ Archivos_Proyectos_Descargar/Imagenes/Cultivos_PDA_A1.jpg

TRD-Tropical Research and Development (1992). Lessons Learned Evaluation conducted for the Selva Economic Revitalization Project. Documento para USAID. Disponible en: http://pdf. usaid.gov/pdf_docs/Xdabf622a.pdf

UNODC-United Nations Office for Drugs Control and Crime (2001). World Drug Report 2000. Oxford: Oxford University Press.

UNODC-United Nations Office on Drugs and Crime (2004). World Drug Report. Volume 2. Statistics. New York: UNODC.

UNODC-United Nations Office on Drugs and Crime (2010). Desarrollo Alternativo en el área andina. Guía técnica. Nueva York: UNODC.

UNODC-United Nations Office on Drugs and Crime (2012). El Modelo de Desarrollo Alternativo de la Región San Martín. Un caso de Desarrollo Económico Local. Lima: UNODC.

UNODC-United Nations Office on Drugs and Crime (2015). Monitoreo de Cultivos de Coca en el Perú 2014. Lima: UNODC.

UNRISD-United Nations Research Institute for Social Development (1994). Illicit Drugs: Social Impacts and Policy Responses. UNRISD Briefing Paper No. 2. World Summit for Social Development. Disponible en: http://www.unrisd.org/80256B3C005BCCF9/(httpAuxPages)/ 4C3D0BE90FAD550480256B6400419B57/\$file/bp2.pdf

USAID-United States Agency for International Development (1986). Ammedment no. five to Project agreement between the Republic of Peru and the United States of America for Upper Huallaga Area Development Project. AID Proyect No. 527-0244.

USAID-United States Agency for International Development (1995). Alternative Development Project. Disponible en: http://www.bvcooperacion.pe/biblioteca/bitstream/123456789/4181/1/ BVCI0003841.pdf

USAID-United States Agency for International Development (2002a). Programa de Desarrollo Alternativo. Resultados 2002. Disponible en: http://www.bvcooperacion.pe/biblioteca/bitstream/123456789/4181/1/BVCI0003841.pdf

USAID-United States Agency for International Development (2002b). Resultados de la verificación de obras y actividades realizadas por el programa de desarrollo alternativo en el 2001. Output verification, abril 2002. Disponible en: http://www.bvcooperacion.pe/biblioteca/bitstream/123456789/1530/1/BVCI0001269.pdf

USAID-United States Agency for International Development (2003). Plan de Desarrollo Alternativo 2003-2006 para el ámbito de San Martín. Disponible en:http://www.bvcooperacion.pe/biblioteca/bitstream/123456789/1529/1/BVCI0001266.pdf

USAID-United States Agency for International Development (2005). Peru Alternative Development Project. Annual Report (April 2004-March 2005). Disponible en: http://www.bvcooperacion.pe/ biblioteca/bitstream/123456789/2636/1/BVCI0002356.pdf

USAID-United States Agency for International Development (2006a) Importancia y efectividad de la estrategia del Programa de Desarrollo Alternativo de USAID/PERÚ. Reporte final. Disponible en: http://pdf.usaid.gov/pdf_docs/PBAAC294.pdf

USAID-United States Agency for International Development (2006b). Programa de Desarrollo Alternativo. Resultados 2006. Disponible en: http://www.bvcooperacion.pe/biblioteca/bitstream/123456789/1511/1/BVCI0001260.pdf

USAID-United States Agency for International Development (2012). USAID/Peru Country Development Cooperation Strategy. Lima: USAID. 